\title{
Le discours religieux des manuels scolaires algériens de l'éducation islamique dans le cycle secondaire
}

Djilali El-Mestari

\section{OpenEdition}

\section{Journals}

Édition électronique

URL : http://journals.openedition.org/trema/2610

DOI : 10.4000/trema.2610

ISSN : 2107-0997

Éditeur

Faculté d'Éducation de l'université de Montpellier

Édition imprimée

Date de publication : 1 décembre 2011

Pagination : $70-80$

ISSN : 1167-315X

Référence électronique

Djilali El-Mestari, « Le discours religieux des manuels scolaires algériens de l'éducation islamique dans le cycle secondaire », Tréma [En ligne], 35 - 36 | 2011, mis en ligne le 01 décembre 2013, consulté le 19 avril 2019. URL : http://journals.openedition.org/trema/2610 ; DOI : 10.4000/trema.2610

Ce document a été généré automatiquement le 19 avril 2019

Trema 
Le discours religieux des manuels scolaires algériens de l'éducation islamique dans le cycle secondaire

Djilali El-Mestari

I. Introduction 
1 Le manuel scolaire constitue un outil pédagogique clé de tout système éducatif. Notre objectif est d'analyser trois manuels d'éducation islamique du cycle secondaire (voir bibliographie), diffusés officiellement en Algérie depuis la réforme appliquée en $2003^{1}$. Nous avons procédé à une analyse qualitative du discours religieux tenant compte des associations thématiques. Ce qui signifie prendre en compte tout discours explicite impliquant une représentation sur l'islam et les valeurs universelles. Dans cette perspective méthodologique, le terme discours est utilisé dans une définition sociale, faisant référence non à des expressions individuelles mais à des systèmes de sens aux conséquences sociales et institutionnelles, selon la conception de Michel FOUCAULT.

2 L'éducation islamique est une discipline qui accompagne l'élève algérien tout au long de son parcours scolaire, de la première année du primaire à la troisième année secondaire, sous deux appellations, "éducation islamique" (terbiyya islamiyya) dans l'enseignement primaire et moyen et «sciences islamiques » (ouloum islamiyya) au niveau secondaire. Son volume horaire est relativement important: une heure et demie par semaine dans le cycle primaire, une heure dans le cycle moyen, et, entre une et deux heures selon les sections dans le cycle secondaire. Pour les cycles primaire et moyen, l'éducation islamique incombe à l'enseignant d'arabe. Dans le cycle secondaire, l'enseignant des «sciences islamiques", formé dans des universités spécialisées en disciplines religieuses, telles l'Université d'El Amir ABDEL KADER de Constantine ou les Facultés des Sciences Islamiques (à Alger, Oran ou Adrar) prend en charge cette discipline. Dans le secondaire, les nouveaux programmes des « sciences islamiques » sont identiques sans aucune différenciation entre les filières littéraires, scientifiques ou techniques.

Les programmes de cette discipline et les contenus des manuels ont été revus à partir de 2003. L'État détermine les orientations générales des programmes à partir des instructions officielles. La Commission nationale des programmes (CNP), composée d'experts de l'éducation (universitaires, inspecteurs et enseignants), définit les grands axes, avant que le Groupe spécialisé des disciplines (GSD) qui réunit des inspecteurs et des enseignants de chaque discipline n'intervienne pour finaliser les nouveaux programmes révisés. Tous les travaux sont présentés à la CNP et validés par le ministère de l'Éducation nationale. Pour ce qui est des nouveaux manuels scolaires, le ministère n'a validé que la collection officielle ${ }^{2}$ éditée par l'Office national des publications scolaires (ONPS) après évaluation des experts de l'Institut national de recherche en éducation (INRE). Le même inspecteur a dirigé l'équipe des auteurs des manuels de sciences islamiques à tous les 
niveaux d'enseignement, du primaire au secondaire. La plupart des auteurs sont membres du GSD, ce qui est le cas aussi pour les autres disciplines.

\section{Le cadre idéologique des programmes des "sciences islamiques »}

3 La révision des programmes de l'éducation islamique s'inscrit dans le cadre des principes constitutionnels qui déterminent la mission de l'école algérienne, invitée à répondre à des défis et à faire face aux enjeux définis par la loi d'orientation de l'Éducation nationale. Ces éléments constituent pour le système éducatif le cadre idéologique de référence et fixent au discours religieux les fondamentaux à inculquer aux élèves. Les principes constitutionnels comprennent trois dimensions :

(1) La dimension nationale et ses référents identitaires : l'islamité, l'arabité et l'amazighité ;

(2) La dimension démocratique avec pour corollaires l'égalité des chances pour tous les élèves, indépendamment de la région géographique ou du statut social, ainsi que la nécessité d'intégrer l'exercice de la démocratie dans les classes par l'implication des élèves dans la vie de l'école ;

(3) La dimension universelle qui doit favoriser une culture scientifique, technique et littéraire en mettant l'accent sur l'ouverture au monde et la formation de l'esprit critique pour permettre à l'élève d'utiliser d'une manière positive les différents savoirs (Loi d'orientation de l'Éducation nationale du 27 janvier 2008, article 2).

Le système éducatif doit ainsi s'adapter aux nouvelles réalités politiques et économiques de l'Algérie et diffuser de nouvelles valeurs telles que les valeurs républicaines et démocratiques, la liberté individuelle et la solidarité sociale, valeurs que souligne l'ordonnance d'avril 1976, révisée par la loi d'orientation de l'Éducation nationale de janvier 2008. Les programmes des «sciences islamiques » sont aussi invités à prendre en compte les défis externes identifiés par la loi d'orientation, à savoir : la mondialisation, les médias et les nouveaux systèmes d'information et de communication, la révolution scientifique et technologique (articles 4, 5 et 6). Ce cadre de référence du système éducatif posé, le contenu des nouveaux manuels des sciences islamiques devra être marqué par cette nouvelle vision fondée sur la prise en compte de l'analyse scientifique, le développement de l'esprit critique, de la créativité et de la responsabilité. Le ministre algérien de l'Éducation nationale a précisé ce réaménagement des enseignements d'éducation islamique en ces termes :

"C'est ainsi que les nouveaux programmes et les nouveaux manuels intègrent une dimension sociale et morale dans l'ensemble du cursus, tout en prenant en considération les besoins des élèves, leur développement psychologique ainsi que leur capacité d'intégration et ce, à tous les cycles d'apprentissage. Cette discipline doit leur permettre, dans les premières années du primaire, l'apprentissage des cinq piliers de l'islam, dans les dernières années du primaire et au moyen, l'intégration progressive des notions liées à la pratique religieuse et des préceptes fondamentaux de la religion musulmane et, à partir de la première année secondaire, l'acquisition d'une culture plus large pour qu'ils puissent faire preuve d'ouverture d'esprit et de tolérance à l'égard des autres religions et cultures » ( BENBOUZID, 2009, p.53-54).

4 Pour autant, ce nouveau discours idéologique est ambivalent et ambigu. Il transmet un message dichotomique entre d'une part l'importance d'une "école mondialisée » ( TOUALBI-THAÂLIBI, 2005 p.24) et «la nécessité d'approfondir l'identité nationale et 
religieuse » (Loi d'orientation, 2008, article 2) d'autre part. Il en est de même dans les programmes des sciences islamiques du secondaire: l'école se voit imposer à la fois de former les citoyens d'une société démocratique et les individus religieux pour une communauté religieuse.

\section{Les thématiques des manuels}

Les manuels du primaire et du cycle moyen abordent des thèmes comme la soumission à la volonté des parents, le respect des personnes âgées et l'amour de la patrie et se rapprochent d'une éducation civique mettant l'accent sur les valeurs morales. Les manuels du secondaire traitent des obligations religieuses, contribuant à une éducation aux pratiques et normes dictées par la chariâ (loi islamique), qui règlent la vie sociale, économique et politique. Cela explique le changement d'appellation. Selon un des auteurs des manuels, l'expression terbiyya islamiya met l'accent sur les acquisitions d'ordre moral, alors que celle de ouloum islamiya, utilisée pour le cycle secondaire, renvoie à l'approfondissement des connaissances en matière d'obligations et de valeurs religieuses.

Les trois manuels de «sciences islamiques » reprennent, de la 1re année secondaire à la Terminale, les mêmes thématiques traitées sous des angles différents.

7 Quel que soit le niveau, la préoccupation majeure des auteurs est d'intégrer les valeurs universelles et humanistes au système des valeurs islamiques, et réciproquement de tirer parti des valeurs religieuses traditionnelles pour construire ou transmettre des valeurs universelles. Les premiers chapitres de chaque manuel abordent essentiellement les croyances, les cultes et les obligations au sein de la famille et de la société, en lien avec les valeurs religieuses traditionnelles. Les chapitres suivants traitent de l'économie, des médias, de l'environnement, de la santé et de l'esthétique, à l'époque actuelle.

Dans le manuel de la 1re année secondaire, cinq chapitres portent sur les valeurs traditionnelles relatives aux enseignements du Coran et du Hadith (paroles du Prophète), en relation avec la vie privée et sociétale de l'apprenant. Il s'agit de la voie du Coran, de la voie de la tradition (la Sunna), quelques études sur la vie du Prophète, les valeurs du culte et de la croyance et les valeurs familiales et sociales. Cinq autres chapitres portent sur des valeurs d'ordre profane, c'est-à-dire selon les titres du manuel, les valeurs rationnelles, les valeurs économiques et financières, les valeurs communicationnelles, les valeurs écologiques ou environnementales, les valeurs artistiques et esthétiques.

Le manuel de la 2e année secondaire reprend et approfondit un certain nombre de questions abordées en 1re année secondaire, comme la voie du Coran, la voie de la tradition, les valeurs de la foi et du culte, les valeurs familiales et sociales et les valeurs communicationnelles. Le manuel aborde aussi, selon ses auteurs, des «sujets importants ». À titre d'exemple, l'introduction comporte des ajouts et des réajustements sur le courant de "l'explication objective " qui va au-delà de "l'interprétation analytique $\|^{3}$ dans l'explication des versets du Coran. Le manuel comprend également des exercices pour une prononciation correcte du Coran. Quelques documents de référence canonique datant de l'époque du Prophète Mohammed et une brève histoire des différents courants et écoles de jurisprudence islamique sont proposés à l'apprenant. Ce manuel évoque pour la première fois dans l'histoire des manuels scolaires algériens d'éducation islamique la question de l'extrémisme religieux et de son impact sur la société et la pensée religieuse elle-même. Ainsi un paragraphe du chapitre intitulé «Le 
danger de l'extrémisme sur la croyance et la société » précise :

"L'islam rejette l'extrémisme et le considère comme une déviance et un moyen d'affaiblissement de la société musulmane, notamment du lien social et de l'organisation politique » (p.118).

Le manuel de la 3e année secondaire comporte six chapitres aux sujets déjà abordés en 1 re et $2 \mathrm{e}$ années secondaires et reste fidèle à la classification des valeurs signalée. Le dernier chapitre aborde les valeurs du droit et souligne l'importance du respect des Droits Humains dans les relations internationales et la nécessité de respecter les droits des travailleurs ${ }^{4}$, tout en présentant la religion musulmane comme source de tous ces droits. Le manuel inclut dans ses dernières pages onze extraits de textes pour «aider l'enseignant et l'élève à mieux comprendre » les questions à l'étude (p.2). Un seul de ces extraits, le document sur l'accord entre le deuxième Calife des musulmans Omar IBN ALКнАтTAB et le peuple de Palestine (651 J.-C.), est de nature historico-religieuse. Les autres sont d'auteurs contemporains, comme l'extrait de discours du Président de la République lors d'un colloque sur la pensée islamique organisé à Alger en 2002 et intitulé « Nécessité de l'innovation dans l'Islam » (p.183), un article de BEN BADIS ${ }^{5}$ "Le musulman algérien » (p.188) ou un article de Mouloud KASSEM, ministre des Affaires religieuses dans les années soixante-dix, «Identité et authenticité » (p.203). On trouve enfin des extraits d'ouvrages d'auteurs algériens et arabes contemporains, spécialistes en droit international, en psychologie et en histoire. Les textes abordent par exemple les droits de la femme, la santé, l'identité et la modernité, la guerre et la paix au regard de la religion.

\section{Les caractéristiques du discours religieux des manuels}

10 Pour caractériser le discours religieux des trois manuels des "sciences islamiques ", nous nous sommes posé les questions suivantes : sommes-nous devant un discours éducatif et cognitif fondé sur le principe de l'intégration morale ou devant un discours mobilisateur et idéologique? Sommes-nous face à des manuels au contenu religieux pédagogique et objectif, ou face à un discours où la fonction idéologique du religieux l'emporte sur le rationnel et les connaissances avérées?

11 On constate tout d'abord la présence de contenus analogues à ceux qui sont dispensés par les institutions religieuses traditionnelles comme les universités spécialisées dans les disciplines religieuses, les écoles de formation des imams ou les zaouïas, où le cursus religieux comprend les classiques de l'enseignement religieux, c'est-à-dire les études théologiques traitant du Coran, de la Sunna, de la vie du Prophète. Mais, comme l'a fait M. EL AYADI pour le cas du Maroc (2004 p.57), on relève également dans les manuels algériens " un nouveau discours sur la religion" qu'on ne peut classer parmi les contenus des sciences religieuses traditionnelles. Deux constats préliminaires se dégagent du décryptage du discours religieux des manuels scolaires algériens de «sciences islamiques » : un aspect mobilisateur et idéologique, mais aussi une structure ambivalente et sélective. 


\section{Un discours religieux de mobilisation identitaire}

12 Les trois manuels des « sciences islamiques » en usage dans le secondaire donnent l'image d'une religion holiste, qui réglemente non seulement la vie privée mais également la vie économique et sociale ainsi que les pratiques culturelles. Ils vont ainsi jusqu’à présenter la religion comme étroitement liée à la réalité sociale actuelle et interagissant avec les problèmes posés aujourd'hui à l'échelle mondiale. Selon ces manuels, l'islam est en mesure de régler les problèmes individuels, familiaux, économiques et politiques; il contribue au développement, tout en coexistant avec les autres religions et cultures, et englobe toutes les valeurs produites par l'expérience humaine dans l'ère moderne. Ainsi des chapitres «Les valeurs dans le Coran » (p.25-34) ou « Le travail et la production dans l'islam et le problème du chômage» (p.40) du manuel de 1re année secondaire. Autre exemple, dans le manuel de la 3e année secondaire, le chapitre «Les Droits de l'Homme dans les relations publiques et le droit international " signale les progrès liés à la Déclaration Universelle des Droits de l'Homme de 1948, tout en soulignant que «l'islam connaît tous ces droits depuis plus de 1400 ans » (p.162).

Les manuels entretiennent ainsi une image idéale des valeurs religieuses et défendent un système de représentation culturel et identitaire où "la culture sacrée fait l'objet d'opérations didactiques importantes" (TOUALBI-ThAÂLIBI, 2005, p.24). Même lorsqu'il s'agit des valeurs modernes à contenus économique, politique, communicationnel ou esthétique, l'on invoque la notion de "spécificité » et l'on distingue valeurs « islamiques » et valeurs modernes "non-islamiques ». Ce type d'analyse transparait dans les chapitres tels que « les droits et les libertés civiques en islam » (2e A.S, p.33-35), «Les valeurs dans le Coran » (1re A.S. p.25-34) ou "l'invasion culturelle et ses dangers pour les sociétés » (2e A.S, p.44-45). Le discours des manuels scolaires algériens d'éducation religieuse est ainsi fidèle à l'esprit « salafiste » (réformisme prônant le retour aux sources premières), malgré l'absence d'extraits d'ouvrages de réformistes musulmans, en dehors de l'article de BEN BADIS dans le manuel de 3e année secondaire.

\section{Un discours religieux ambivalent et sélectif}

Outre cet aspect idéologique et mobilisateur chargé d'émotions, le discours religieux s'avère à la fois ambivalent et sélectif, notamment dans la question des relations de l'islam avec les autres religions et cultures. Plusieurs chapitres mettent en avant les valeurs de fraternité, d'amitié, de liberté et de tolérance, comme en témoigne l'intitulé "L'Islam et la coexistence pacifique " (1re A.S. p.147-151). Autre exemple, dans "Les droits et les libertés civiques en Islam» (2e A.S. p.33-35), il est fait état des bonnes relations du Prophète avec les autres religions: "La preuve est qu'il s'est marié à une juive Rayhana » (p.35). Ou encore, dans «L'islam et les religions monothéistes », il est écrit à propos de celle-ci que « les messages divins constituent un ensemble cohérent et une substance commune et constante, l'Unicité de Dieu » (3e A.S. p.350). La fraternité des prophètes des trois religions est rappelée par cette phrase : « les adeptes des ces religions (doivent) rester frères et loin de tout conflit entre eux » (3e A.S. p.58). Dans « Les rapports sociaux entre les musulmans et les non-musulmans » (3e A.S, p.146), on insiste sur la tolérance des différences, notamment religieuses, et le respect des droits des nonmusulmans en pays musulman. L'accent est mis sur « les droits de la protection, la liberté 
de la pratique religieuse, le droit au travail et même le droit d'exercer des fonctions supérieures au sein de l'Etat musulman»(p.149). Il en est de même dans "La reconnaissance du pluralisme culturel, politique et religieux » (3e A.S, p.170) et « la paix dans monde est l'objectif ultime de la théorie islamique des relations internationales » (3e A.S. p.170).

14 Toutefois, parallèlement à cet appel à la tolérance, s'observe dans le même discours une tendance inverse. Dans le chapitre «l'invasion culturelle et ses dangers à l'égard des sociétés » (2e A.S, p. 44) un texte voit dans les traditions des autres religions monothéistes chrétienne et judaïque une menace pour les croyants de l'islam.

En outre, le discours des manuels d'instruction religieuse du secondaire est un discours sélectif. Cette démarche vise à justifier la logique de vénération et de sacralisation, notamment dans les chapitres relatifs à la relation entre les valeurs religieuses de l'islam et les valeurs modernes. Cette instrumentalisation de l'héritage religieux est présente dans la plupart des chapitres. À titre d'exemple, certains traitent des questions d'actualité en utilisant des versets coraniques ou des paroles du Prophète, sans tenir compte des contextes de leur énoncé et ce, pour justifier l'image d'un islam en mesure de régler les problèmes actuels. Dans le chapitre «l'importance de la solidarité pour la protection de l'environnement » (1re A.S. p.161), l'interdiction de cracher par terre prônée par le Prophète est rattachée à la lutte contre la pollution. Le manuel de la 2e année secondaire explique, dans un chapitre intitulé «le phénomène démographique dans le Saint Coran ", l'importance de cette question dans la religion islamique en utilisant des versets coraniques sans relation avec le contexte actuel. On trouve aussi certains chapitres comme " développement humain entre quantité et qualité » (2e A.S p.28) avec des soustitres comme "le Coran et les processus démographiques» ou "le Coran et le développement durable ", tendant à démontrer que le discours coranique a déjà évoqué ces sujets dits modernes.

Ce mode de fonctionnement est confirmé par la façon de traiter le courant Ibâdite. Dans le chapitre «histoire des écoles de jurisprudence» (2e A.S p.84), ce courant est présenté comme une école de jurisprudence et non comme une doctrine théologique. Les auteurs des manuels, tolérants avec le pluralisme des écoles islamiques de jurisprudence, ne le sont pas avec le pluralisme des écoles théologiques en islam. En outre, nous constatons l'absence du discours mystique dans tous les manuels du secondaire, bien que le soufisme populaire soit un phénomène réel dans la société algérienne. De même les manuels ne contiennent aucun chapitre sur l'école des chiites.

\section{Pour conclure}

Le discours religieux des manuels scolaires algériens de l'éducation islamique du cycle secondaire est un discours mobilisateur basé d'une part, sur une logique de vénération et de sacralisation et d'autre part, sur une logique sélective et ambivalente qui demeure marquée par l'apologétique. Ces caractéristiques sont dues aux mécanismes de défense des valeurs religieuses contre un ennemi supposé comme le démontrent les titres et les contenus des différents chapitres des manuels.

Outre les problèmes d'ordres pédagogique et éducatif que pose le discours mobilisateur, les éléments de "sacralisation ", notamment en abordant des connaissances modernes, engendrent des problèmes d'ordre cognitif, comme l'absence de clarté sur le plan 
épistémologique quand il s'agit de prendre en charge des questions importantes dans l'enseignement religieux telles que celles de la croyance et du savoir, des limites entre l'universel et le local dans la culture religieuse, de la différence entre mythe et histoire, et de la différence entre la morale et le savoir (ISMAIL 2004 p.95-96). Cette sacralisation amplifie l'éloignement problématique entre l'enseignement de l'éducation islamique et d'autres disciplines, en instaurant une absence de complémentarité et une incohérence entre les disciplines religieuses et celles traitant du savoir pratique des sciences nonreligieuses. Ce discours est un discours de ségrégation entre le savoir sur le présent et celui portant sur le passé (le savoir religieux). Le savoir issu du passé devient une source absolue et déterminante de la connaissance du présent, ce qui renforce un discours idéologique du religieux qui ne permet pas à l'apprenant de faire la distinction entre la religion comme foi et son instrumentalisation idéologique.

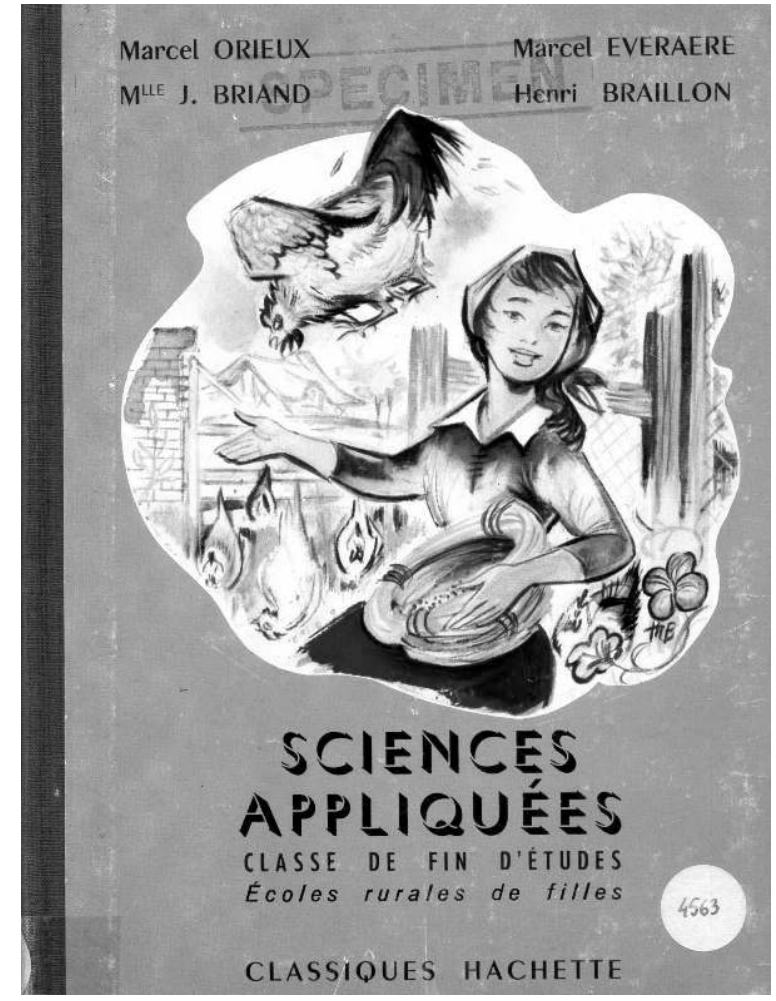

Sciences appliquées, classe de fin d'études, écoles rurales de filles, M. ORIEUX, J. BRIAND, M. EVERAERE, H. BRAILLON, Paris : Hachette, 1959, 205 p., p. 10 - Couverture - Source : CEDRHE.

\section{BIBLIOGRAPHIE}

BENBOUZID B., La réforme de l'éducation en Algérie, enjeux et réalisations, Alger, Casbah éditions, 2009

CHARFI M., La Réforme du système éducatif en Tunisie, Actes d'une conférence, in Transition et perspectives, éditions Institut national d'études de stratégie globale, Algérie, 2000

EL AYADI M., L'école publique moderne et l'enseignement religieux, in Coll. Enseigner la religion aujourd'hui ?, Actes de colloque, Fondation du Roi ABDU-AzIZ et la Fondation Konrad ADENAUER Casablanca, 2004 
GHALEM M., Idéologie et histoire : Le livre d'Histoire de la 2e année secondaire, in GHALEM M. et REMAOUN H. (dir), Comment enseigner l'Histoire en Algérie, CRASC, Algérie (en langue arabe), 1995 GHALEM M., Philosophie et école, lecture sur les propositions de la commission nationale de la réforme du système éducatif, in GHALEM M.et KERROUMI A. (dir), Philosophie, mémoire et institution, CRASC, Algérie,(en langue arabe), 2004

ISMAIL M., L'enseignement religieux : Transmission du savoir ou appel à l'adhésion ?, in ouvrage collectif, Fondation du Roi ABDU-AZIZ et la Fondation Konrad ADENAUER, Le manuel scolaire dans le système éducatif algérien, réalité et perceptives. Actes du colloque des 24-25 novembre 2007, Éditions Centre de recherche scientifique et technique de développement de la langue arabe, Alger, (2008), 2004

REMAOUN H., L'État national et sa mémoire : le paradigme histoire, in REMAOUN-BENGHABRIT N. et HADDAB M. (dir), L' Algérie, 50 ans après. État des savoirs en Sciences Sociales et Humaines (1954-2004), Oran, CRASC, 2008

REMAOUN H., Histoire nationale et pratiques politico-identitaires. Une lecture des manuels officiels en usage dans l'école algérienne, in MAROUf N. et CARPENTIER C. (dir), École langue, identités, Paris, l' HARMATTAN (Version en langue arabe, in Insaniyat, n³ ,1997), 1997

REMAOUN H., L'école, histoire et enjeux institutionnels dans l'Algérie indépendante, in Les temps modernes, $\mathrm{n}^{\circ} 580,1995$

Revue Prologues, (Revue maghrébine du livre), trimestrielle $n^{\circ} 21$. Quel avenir pour l'éducation au Maroc ? À propos de la Charte nationale de l'éducation et de la formation, contributions de BENMOKHTAR R., Boukous A., CHEDdAdi A., El AYAdI M., Esmili H., Mezouar A., Automne 2000, hiver 2001. TOUALBI-THAÂLIBI N., Changement social, représentation identitaire et refonte de l'éducation en Algérie, in TOUALBI-THAÂLIBI N. et TAWIL S. (Dir), L'école à l'épreuve du nouveau monde, Alger (Algérie), Casbah éditions, 2005 TOUALBI-THAÂLIBI N., École, idéologie et droits de l'homme, Alger, Casbah éditions, 2004 TOUALBI-THAÂLIBI N., L'identité au Maghreb. L'errance, Alger, Casbah éditions, 2001

\section{Corpus des manuels des sciences islamiques utilisés (nouveaux programmes)}

(1) SARI M. (Dir), El Mounir fi el Ouloum El Islamiya (Le lumineux dans les sciences islamiques), classe de première année secondaire, Alger, 1re édition,ONPS, 2006

(2) SARI M. (Dir), El Wadih fi el Ouloum El Islamiya (L'évident dans les sciences islamiques) classe de deuxième année secondaire, Alger, 1re édition, ONPS, 2007

(3) SARI M. (Dir), El Moufid fi el Ouloum El Islamiya (L'utile dans les sciences islamiques) terminale, Alger, 1re édition, ONPS, 2008

\section{NOTES}

1. Cette question a été abordée par des chercheurs algériens qui travaillent sur les effets de l'idéologie nationale sur différents manuels scolaires des sciences sociales avant et après la 
réforme du système éducatif. Cf. par exemple : REMAOUN, 1995, 1997, 2008 ; TOUALBI-THAÂLIBI, 2001, 2004 ; GHALEM, 1995, 2004.

2. Initialement le débat a porté sur la possibilité d'ouvrir le marché de l'édition scolaire à des maisons d'éditions privées, pour une plus grande diversité des contenus.

3. L'interprétation analytique se réfère dans toute explication des versets coraniques aux textes sacrés, tandis que l'explication objective peut se référer à la raison et à la science, sans se limiter exclusivement aux textes sacrés. Voir l'introduction du manuel des sciences islamiques de 2e A.S, signée par Moussa SARI (coordinateur du manuel).

4. Comme le droit à un salaire pour toute activité exercée, le droit aux revendications et au recours à la Justice, ainsi que le droit aux réparations en cas de préjudice (Manuel de la 3e A.S p. 174 à 178).

5. Abdelhamid BEN BADIS est précurseur du réformisme (islah) algérien et fondateur en 1931 de l'association des Ulémas musulmans algériens.

\section{RÉSUMÉS}

Des recherches ont ouvert le débat sur la nature, le contenu et les objectifs de l'enseignement religieux à l'école d'un certain nombre de systèmes éducatifs contemporains au Maghreb. Notre objectif consiste à analyser le discours religieux diffusé dans l'école algérienne, à partir de trois manuels scolaires d'éducation religieuse du cycle secondaire, en prenant en considération les réformes du système éducatif algérien depuis 2003. Nous tenterons de répondre à la question suivante: le discours religieux des manuels scolaires d'éducation islamique reflète-t-il une représentation cognitive sur l'islam ou renvoie-t-il à un discours idéologique et identitaire?

Some research has raised the debate about the nature, content and even the targets of the religious education of some contemporary educational systems in the Maghreb. Our aim is to analyze the religious content of the speech distributed through Algerian schools from three textbooks of religious education in secondary schools, taking into account the new reforms of the Algerian education system since 2003. We try to answer the following question: Does the religious discourse of Islamic education textbooks reflect a cognitive representation of Islam or does it refer to an ideological and identity discourse?

\section{INDEX}

Mots-clés : Algérie, discours religieux, éducation islamique, enseignement secondaire, idéologie, manuel scolaire

Keywords : Algeria, ideology, islamic education, religious discourse, secondary school, textbook

\section{AUTEUR}

\section{DJILALI EL-MESTARI}

CRASC, Oran, Algérie 